\title{
Dysphagie (Schluckstörung)
}

\section{Schluckstörungen ziehen erhebliche Beeinträchtigungen des körperlichen, psychischen und sozialen Wohlbefindens nach sich und bedürfen immer einer Abklärung}

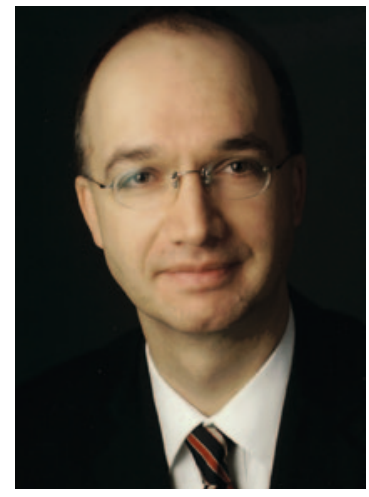

Ao. Univ. Prof. Dr. Dietmar Thurnher Stv. Leiter der klinischen Abteilung für allgemeine HNO

Leiter des interdisziplinären Tumorboards für Kopf- und Halstumoren

Univ. Klinik für Hals-Nasen-Ohrenkrankheiten Medizinische Universität Wien

Schluckstörung oder Dysphagie bezeichnet alle subjektiven und objektiven Hindernisse bei der Aufnahme oder beim Weitertransport von Nahrung, bedingt z. B. durch Schluckwiderstand, Hochwürgen von Speisen und Schmerzen beim Schlucken. Das Spektrum der zugrunde liegenden Erkrankungen kann von völlig harmlosen Ursachen (psychisches Fremdkörpergefühl) bis zu lebensgefährlichen Karzinomen des des Kopf-Hals-Bereiches reichen und erfordert deshalb eine sofortige Abklärung der Schluckstörung. (häufige Ursachen entnehmen Sie bitte der MindMap, Abb. 1)

Anamnestisch geben PatientInnen ein Würgen während des Schluckaktes und Hochwürgen von bereits verschluckter Nahrung an. Daneben kommt es zu Husten und Aspiration von Nahrung in die unteren Atemwege. Ausdruck einer

\footnotetext{
Ao. Univ. Prof. Dr. Dietmar Thurnher Stv. Leiter der klinischen Abteilung für allgemeine $\mathrm{HNO}$

Leiter des interdisziplinären Tumorboards für Kopf- und Halstumoren

Univ. Klinik für Hals-Nasen-Ohrenkrankheiten Medizinische Universität Wien,

Währinger Gürtel 18-20, 1090 Wien
}

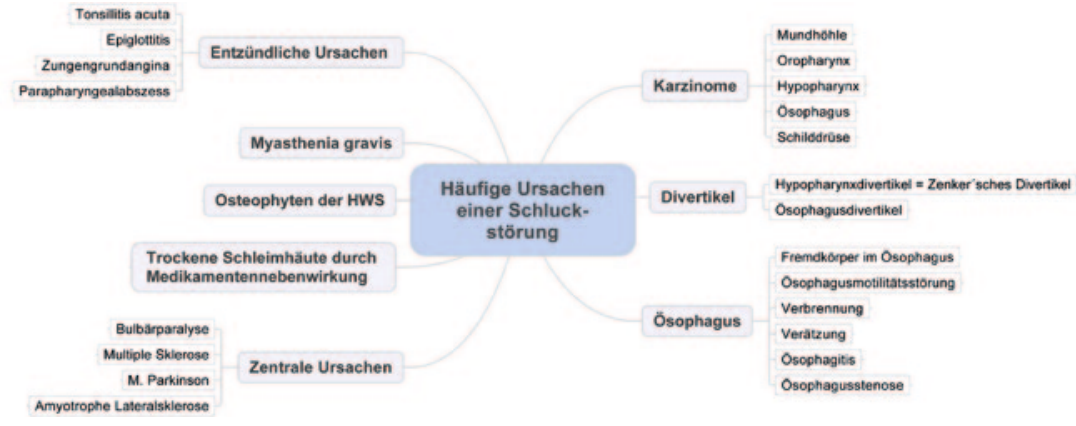

Abb. 1. Mindmap Häufige Ursachen einer Schluckstörung

chronischen Dysphagie sind Mangelernährung und Exsikkose der betroffenen Person.

\section{Diagnose}

Diagnostisch wird ein kompletter HNO-Status und ein Schluckröntgen, weiterführend auch eine CT oder MRT der Kopfund Halsregion durchgeführt. Bei Verdacht auf ein Malignom im oberen Aerodigestivtrakt muss zur Ausdehnungsbestimmung und Probegewinnung eine Panendoskopie in Allgemeinnarkose durchgeführt werden.

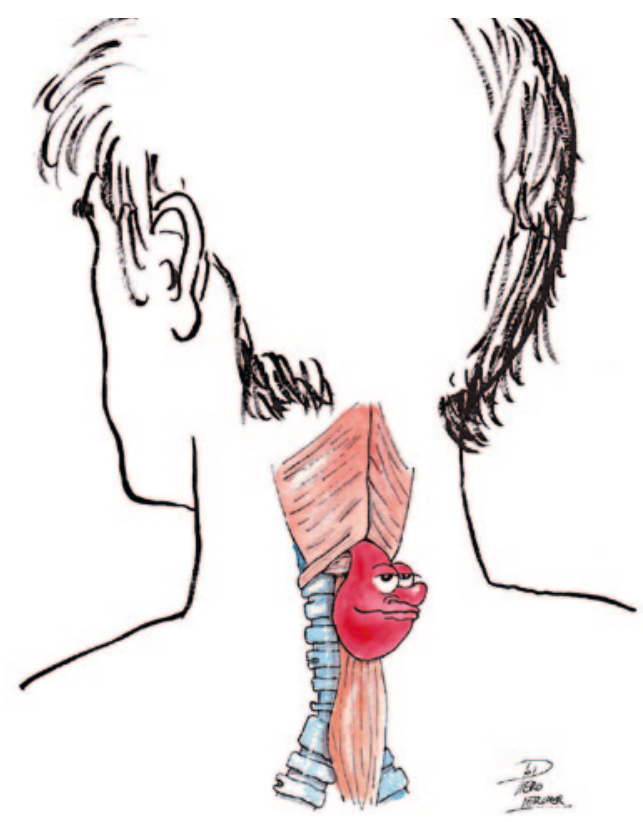

\section{Therapie}

Abb. 2: Zenker'sches Divertikel, credit Piero Lercher aus: Thurnher, Grasl, Erovic, Lercher. HNO-Heilkunde. Springer, 1. Auflage 2011

Therapeutisch steht die Behandlung der Grunderkrankung, also z. B. Operation eines Zenker-Divertikels ( $A b b$. 2) oder die Behandlung eines zugrundeliegenden Morbus Parkinson im Vordergrund.

Zusätzlich wird ein logopädisches Schlucktraining durchgeführt.

Bei nicht behandelbaren Grunderkrankungen dient die Schluckrehabilitation der Verhinderung von Aspirationen und einer Sicherstellung der Ernährung des Patienten.
Ao. Univ. Prof. Dr. Dietmar Thurnher

Stv. Leiter der klinischen Abteilung für allgemeine HNO

Leiter des interdisziplinären Tumorboards für

Kopf- und Halstumoren
Univ. Klinik für Hals-Nasen-Ohrenkrankheiten

Medizinische Universität Wien

Währinger Gürtel 18-20

1090 Wien

$\mathrm{T}:+43140400428$

Privatordination

Privatklinik Confraternität

Skodagasse 32

1080 Wien

W:airwaymanagement.at

W:headandneckcancer.at

\section{Korrespondenz:}

Tel.: 01 / 4011 4- 5701 